\title{
ORIGINAL
}

\section{Long-term empagliflozin therapy improves levels of hepatic fibrosis marker in patients with non-alcoholic fatty liver disease complicated by type 2 diabetes mellitus}

\author{
Satoshi Shinozaki ${ }^{1,2}$, Toshiyuki Tahara ${ }^{3}$, Alan Kawarai Lefor ${ }^{4}$, and Masahito Ogura ${ }^{5}$ \\ ${ }^{1}$ Shinozaki Medical Clinic, Tochigi, Japan, ${ }^{2}$ Department of Medicine, Division of Gastroenterology, Jichi Medical University, Tochigi, Japan, \\ ${ }^{3}$ Saiseikai Utsunomiya Hospital, Tochigi, Japan, ${ }^{4}$ Department of Surgery, Jichi Medical University, Tochigi, Japan, ${ }^{5}$ Department of Diabetes, \\ Endocrinology and Nutrition, Graduate School of Medicine, Kyoto University, Kyoto, Japan
}

\begin{abstract}
The long-term outcomes of patients with non-alcoholic fatty liver disease (NAFLD) treated with sodium-glucose cotransporter-2 inhibitors remain indeterminate. Empagliflozin improves hyperglycemia by increasing glucose excretion in the urine, and it reduces fat volume and insulin resistance. The aim of this study is to assess the effect of long-term empagliflozin therapy on hepatic inflammation, function and fibrosis in patients with NAFLD. This is a two-center retrospective observational study including patients with NAFLD complicated by type 2 diabetes mellitus. We retrospectively reviewed the medical records. Changes in parameters were investigated over one-year empagliflozin treatment. Twenty-four patients treated with empagliflozin were evaluated. Weight, body mass index, aspartate aminotransferase, alanine aminotransferase, gamma-glutamyl transpeptidase, fasting plasma glucose, hemoglobin A1c, serum insulin and homeostasis model assessment insulin resistance significantly decreased during treatment $(p<0.05)$. Albumin-bilirubin (ALBI) score, a marker of hepatic function, was significantly improved $(p<0.01)$. The FIB-4 index and Mac-2 Binding Protein Glucosylation Isomer, markers of hepatic fibrosis, significantly improved $(p<0.01)$. One-year empagliflozin treatment of patients with NAFLD complicated by type 2 diabetes mellitus significantly improves markers of hepatic inflammation, function and fibrosis. J. Med. Invest. 67:280-284, August, 2020
\end{abstract}

Keywords : non-alcoholic fatty liver disease, non-alcoholic steatohepatitis, sodium-glucose transporter 2 inhibitors, diabetes mellitus, hepatitis

\section{INTRODUCTION}

Non-alcoholic fatty liver disease (NAFLD) is a major complication of type 2 diabetes mellitus. NAFLD is a hepatic manifestation of insulin resistance and metabolic syndrome, and frequently complicates metabolic diseases such as diabetes, dyslipidemia, obesity and hypertension. NAFLD is grossly classified as non-alcoholic fatty liver (NAFL) and non-alcoholic steatohepatitis (NASH). The former has only fat deposition, and the latter is characterized by fibrosis due to recurrent necrosis and inflammation (1). NASH is a major risk factor for the development of cirrhosis and hepatocellular carcinoma. The prevalence of NASH is about $3-5 \%$ worldwide and $1-2 \%$ in Japan (1, 2). In subjects with type 2 diabetes mellitus, a high prevalence of NAFLD (65\%) and NASH (7\%) were reported (3), and type 2 diabetes and obesity are independent predictors of NASH (4). Therefore, controlling the inflammation and fibrosis associated with NAFLD in patients with type 2 diabetes is important to prevent the development of cirrhosis and hepatocellular carcinoma. Since Japanese people are more prone to develop fatty liver than non-Hispanic whites in the United States even in people with a slightly elevated body mass index (BMI) (5), the therapeutic strategy for Japanese people with NAFLD is quite important.

Empagliflozin, a sodium-glucose cotransporter 2 (SGLT2) inhibitor, improves hyperglycemia by increasing glucose excretion

Received for publication March 29, 2020 ; accepted May 26, 2020.

Address correspondence and reprint requests to Satoshi Shinozaki, M.D., Ph.D., Shinozaki Medical Clinic, 6-1-13 Kiyoharadai, Utsunomiya, Tochigi, 321-3223, Japan and Fax : +81-28-667-7305. in the urine. It was also reported to reduce fat volume and improve insulin resistance $(6,7)$. In a murine model of NASH, empagliflozin prevented the development of NASH by decreasing inflammation and collagen deposition (8). In the EMPA-REG OUTCOME trial, while alanine aminotransferase (ALT) was significantly reduced, the diagnosis of NAFLD was not established in that study (9). It has been reported that short-term SGLT2 inhibitor treatment results in improvement of inflammatory markers of NAFLD and fat deposition (10-12). Few studies reported long-term outcomes of patients with NAFLD treated with an SGLT2 inhibitor. The aim of this study is to assess the long-term effect of empagliflozin on hepatic inflammation, function and fibrosis in patients with NAFLD complicated by type 2 diabetes mellitus.

\section{METHOD}

\section{Study population and inclusion}

This is a two center retrospective observational study including patients with NAFLD complicated by type 2 diabetes mellitus. We retrospectively reviewed their medical records and abstracted the following data : age, gender, smoking habits, alcohol consumption, combination of medications, complications, weight and laboratory data.

The inclusion criteria for this study were : (1) NAFLD complicated by type 2 diabetes mellitus treated with empagliflozin, (2) presence of hepatorenal echo contrast on abdominal ultrasound, (3) persistent elevation of ALT $>30$ for more than three months before starting empagliflozin, (4) negative hepatitis B surface antigen and hepatitis $\mathrm{C}$ virus antibody, (5) normal serum immunoglobulin-G level, (6) alcohol consumption $<30 \mathrm{~g} /$ day in males 
and $<20 \mathrm{~g} /$ day in females. We excluded patients with severe chronic kidney disease (serum creatinine $>2.5$ ), those who stopped taking empagliflozin within one year or those missing required laboratory data. From January 2017 to August 2018, patients diagnosed with NAFLD complicated by type 2 diabetes mellitus and treated with empagliflozin at the Saiseikai Utsunomiya Hospital or the Shinozaki Medical Clinic were included. The institutional review boards at each center approved this retrospective review.

\section{Evaluation of hepatic inflammation, function and fibrosis}

Although liver biopsy is the best method to evaluate the degree of hepatic inflammation and fibrosis, it is difficult to perform in routine clinical practice because of its complexity and risk of serious adverse events. Hepatic inflammation was evaluated with serum ALT levels, a validated marker for progression of hepatic fibrosis in patients with NASH $(13,14)$. Hepatic function was estimated with the albumin-bilirubin (ALBI) score, calculated using both serum albumin and bilirubin levels and reflects indocyanine green retention rate at $15 \mathrm{~min}$ (ICG-R15) (15). The degree of hepatic fibrosis was estimated using the cutoff index of the serum Mac-2 Binding Protein Glucosylation Isomer (M2B$\mathrm{PGi}$ ) that is a glycoprotein biomarker reflecting the grade of hepatic fibrosis and a predictor of developing hepatocellular carcinoma over the long-term (16-18). The FIB-4 index and NAFLD fibrosis score were used as markers of hepatic fibrosis $(19,20)$. The degree of insulin resistance that is closely associated with pathogenesis of NAFLD was evaluated with HOMA-IR (homeostasis model assessment insulin resistance) (21).

\section{Statistical analysis}

Statistical analysis was carried out using StatFlex 7.0 software (Artech Co., Ltd., Osaka, Japan). Differences were considered significant with $p<0.05$. The chi-square goodness-of-fit test did not show a normal distribution of the values of clinical parameters. Changes in clinical parameters were evaluated with the Wilcoxon rank-sum test. Correlation was analyzed using Spearman's Rank correlation coefficient.

\section{RESULTS}

\section{Baseline characteristics}

From January 2017 to August 2018, 28 patients were treated with empagliflozin. Twenty-four of these 28 patients were included in the final analysis after excluding four patients for the following reasons : empagliflozin was stopped within one year because the patient did not visit the clinic $(n=2)$ and a lack of required laboratory data $(n=2)$.

Characteristics of the 24 patients treated with empagliflozin $10 \mathrm{mg}$ daily for one year are shown in Table 1. More than half of the patients were treated with a statin drug for hypercholesterolemia, and there were no further additions of statins after starting empagliflozin. Tocopherol or ursodeoxycholic acid was not used during the study period. The dose of empagliflozin remained at $10 \mathrm{mg}$ once daily for one year without dose escalation. There were no dose escalations or cession of anti-diabetic drugs except in one patient who underwent dose escalation of metformin from $1500 \mathrm{mg}$ to $2000 \mathrm{mg}$ per day. No adverse events were observed.

To understand the difference among the hepatic fibrosis markers before starting empagliflozin, we investigated the correlation between M2BPGi and markers of hepatic fibrosis. Significant positive correlations with M2BPGi were observed with the FIB-4 index $(r=0.691, p<0.001)$ and the NAFLD fibrosis score $(\mathrm{r}=0.644, \mathrm{p}<0.001)$
Improvement of markers of hepatic inflammation, function and fibrosis during one-year empagliflozin treatment

Changes in clinical parameters were investigated after oneyear empagliflozin treatment (Table 2). Weight, BMI, AST, ALT and $\gamma$-GTP significantly decreased, and lipid parameters did not significantly change after one year of treatment. Diabetic markers including fasting plasma glucose (FPG), hemoglobin A1c (HbA1c), serum insulin and HOMA-IR significantly improved.

Table 1. Baseline characteristics of patients treated with empagliflozin

\begin{tabular}{|l|c|}
\hline & $\mathrm{N}=24$ \\
\hline Age, years, mean \pm standard error & $57.4 \pm 2.9$ \\
\hline Gender, male, $\mathrm{n}$ & $15(63 \%)$ \\
\hline Current Smoker, $\mathrm{n}$ & $7(29 \%)$ \\
\hline Complications treated with medication, $\mathrm{n}$ & \\
Dyslipidemia & $15(63 \%)$ \\
Hypertension & $11(46 \%)$ \\
Gastroesophageal reflux disease & $9(38 \%)$ \\
Hyperuricemia & $3(13 \%)$ \\
\hline Combination use, $\mathrm{n}$ & \\
Statins & $13(54 \%)$ \\
Angiotensin II receptor blockers & $9(38 \%)$ \\
Metformin & $8(33 \%)$ \\
DPP-4 inhibitors & $6(25 \%)$ \\
Pioglitazone & $3(13 \%)$ \\
Bezafibrate & $2(8 \%)$ \\
GLP-1 analog & $1(4 \%)$ \\
Ezetimibe & $1(4 \%)$ \\
\hline
\end{tabular}

DPP-4 : dipeptidyl peptidase-4, GLP-1 : glucagon-liken peptide-1

Table 2. Changes during one-year empagliflozin therapy

\begin{tabular}{|l|c|c|c|}
\hline & Before & One-year & p-value \\
\hline Weight, kg, mean $\pm \mathrm{SE}$ & $82.2 \pm 4.7$ & $78.8 \pm 4.5$ & $<0.001$ \\
\hline Body mass index & $30.3 \pm 1.3$ & $28.9 \pm 1.2$ & $<0.001$ \\
\hline Platelet, x10 $/ \mu \mathrm{L}$ & $22.1 \pm 1.4$ & $22.4 \pm 1.3$ & 0.334 \\
\hline AST, U/L & $45.7 \pm 4.2$ & $33.4 \pm 3.7$ & $<0.001$ \\
\hline ALT, U/L & $53.7 \pm 4.5$ & $35.3 \pm 4.5$ & $<0.001$ \\
\hline ALP, U/L & $226.9 \pm 11.9$ & $216.5 \pm 14.6$ & 0.072 \\
\hline$\gamma$-GTP, U/L & $72.3 \pm 8.7$ & $50.5 \pm 6.4$ & $<0.001$ \\
\hline Total bilirubin, $\mathrm{mg} / \mathrm{dL}$ & $0.85 \pm 0.07$ & $0.78 \pm 0.08$ & 0.095 \\
\hline LDL cholesterol, $\mathrm{mg} / \mathrm{dL}$ & $104.8 \pm 7.1$ & $98.5 \pm 5.5$ & 0.481 \\
\hline HDL cholesterol, $\mathrm{mg} / \mathrm{dL}$ & $46.6 \pm 2.5$ & $49.6 \pm 2.3$ & 0.087 \\
\hline Triglyceride, $\mathrm{mg} / \mathrm{dL}$ & $256.9 \pm 68.6$ & $244.2 \pm 43.9$ & 0.404 \\
\hline Serum albumin, $\mathrm{g} / \mathrm{dL}$ & $4.3 \pm 0.1$ & $4.5 \pm 0.1$ & 0.002 \\
\hline Estimated GFR, $\mathrm{ml} / \mathrm{min} / 1.73 \mathrm{~m}^{2}$ & $81.6 \pm 3.2$ & $79.0 \pm 3.0$ & 0.092 \\
\hline Fasting plasma $\mathrm{glucose}, \mathrm{mg} / \mathrm{dL}$ & $149.9 \pm 9.7$ & $115.2 \pm 5.0$ & $<0.001$ \\
\hline Serum insulin, $\mu \mathrm{U} / \mathrm{mL}$ & $18.0 \pm 4.0$ & $11.8 \pm 2.3$ & 0.009 \\
\hline HbA1c, $\%$ & $7.3 \pm 0.2$ & $6.7 \pm 0.1$ & $<0.001$ \\
\hline HOMA-IR & $6.5 \pm 1.3$ & $3.1 \pm 0.5$ & $<0.001$ \\
\hline
\end{tabular}

$\mathrm{SE}:$ standard error, AST : aspartate aminotransferase, ALT : alanine aminotransferase, ALP : alkaline phosphatase, $\gamma$-GTP $\gamma$-glutamyl transpeptidase, LDL : low density lipoprotein, HDL : high density lipoprotein, GFR : glomerular filtration rate, HbA1c : hemoglobin A1c (glycated hemoglobin), HOMA-IR : homeostasis model assessment insulin resistance 
The levels of markers of hepatic function and fibrosis are shown in Figure 1. The ALBI score, a marker of hepatic function, was significantly improved. Hepatic fibrosis markers including the FIB-4 index and M2BPGi significantly decreased at 12 months. The NAFLD fibrosis score had a likelihood of a decrement. Unlike other parameters, M2BPGi showed a significant difference at 12 months but not at six months compared to the baseline value.
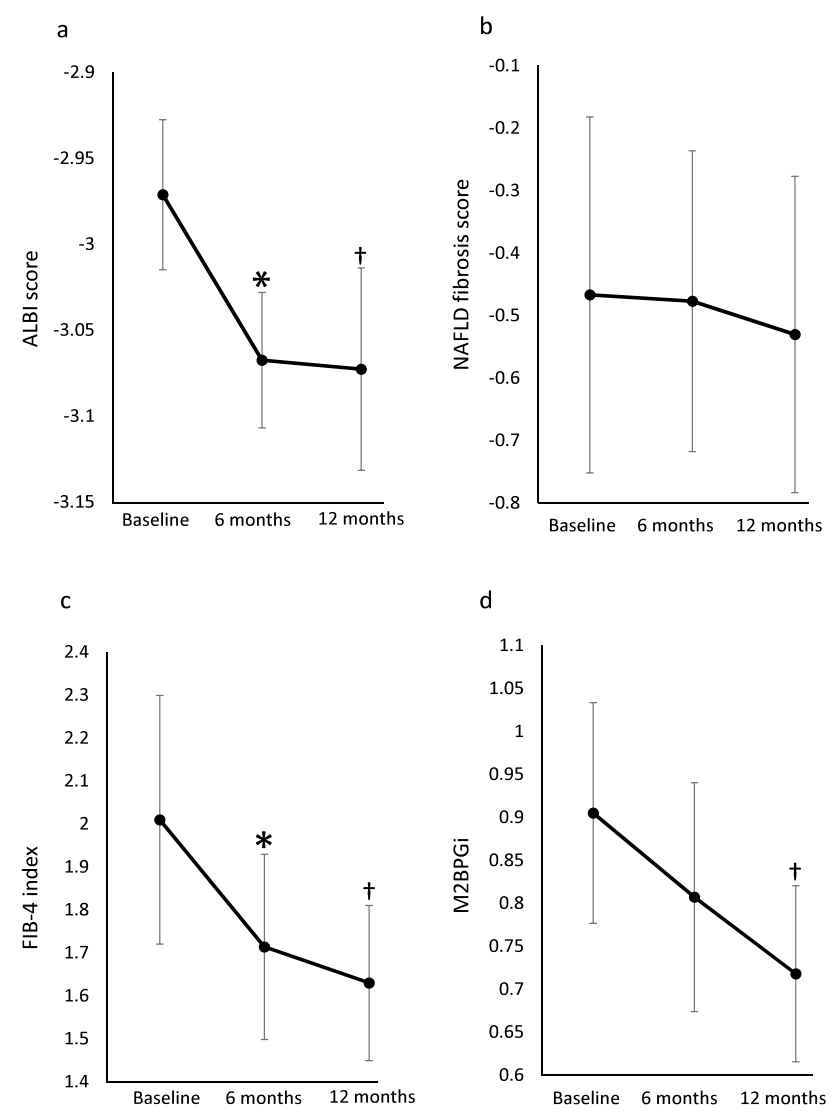

Figure 1. Changes in parameters for hepatic function and fibrosis $* p<0.05, \dagger p<0.01$. a) Albumin-bilirubin (ALBI) score, b) NonAlcoholic Fatty Liver Disease (NAFLD) fibrosis score, c) FIB-4 index and d) cutoff index of the serum Mac-2 Binding Protein Glucosylation Isomer (M2BPGi).

\section{DISCUSSION}

This observational study demonstrates the effectiveness of a one-year course of empagliflozin $10 \mathrm{mg}$ daily to improve markers of hepatic inflammation, function and fibrosis in patients with NAFLD complicated by type 2 diabetes mellitus. One-year empagliflozin treatment improved weight, plasma glucose and insulin resistance. Since the observation period in previous studies reporting the effectiveness of SGLT2 inhibitors on NAFLD was at most six months, the observation period in the present study is comparatively long. This is the first study demonstrating an improvement in markers of hepatic fibrosis due to NAFLD after one-year treatment with empagliflozin.

Empagliflozin ameliorates hepatic steatosis, inflammation and fibrosis in a murine model of NASH and diabetes mellitus (8). The clinical efficacy of empagliflozin on NAFLD has been reported. The EMPA-REG OUTCOME trial revealed a significant decrease in serum liver enzyme levels including AST and ALT in the empagliflozin-treated group compared with the placebo group (9). However, the diagnosis of NAFLD was not established in the trial. The E-LIFT trial from India demonstrated that treatment with empagliflozin significantly reduced liver fat and ALT compared to placebo during a 20 week treatment period evaluated by MRI-derived proton density fat fraction (11). A randomized controlled trial from Germany reported a significant reduction of liver fat content in the empagliflozin treated group compared with the placebo group, and this trial did not show improvement in LDL cholesterol levels over 24 weeks (22). A single-arm pilot study with empagliflozin $25 \mathrm{mg}$ daily for 24 weeks demonstrated improvements in steatosis, ballooning and fibrosis seen on liver biopsy (23). The present study has the longest observation period in patients with NAFLD treated with empagliflozin.

A recent Japanese study including 20 patients with NAFLD complicated by type 2 diabetes mellitus treated with canagliflozin for one year reported a significant reduction in weight and ALT without improving the FIB-4 index (24). In the present study, the FIB-4 index and M2BPGi were significantly improved. The FIB-4 index before starting empagliflozin in the present study $(2.0 \pm 1.4)$ is somewhat higher than in the canagliflozin study (1.2 \pm 0.5$)(24)$. The difference in the change of the FIB-4 index may be explained by the degree of hepatic fibrosis before starting treatment and/or the type of SGLT2 inhibitor. A recent Japanese randomized controlled trial using luseogliflozin versus metformin reported that patients in the luseogliflozin group had a significant reduction of liver fat deposition (25). A recent Japanese NAFLD study with luseogliflozin reported that there was no significant reduction of M2BPGi during 24 weeks of treatment (26). The present study also did not show a significant reduction of M2BPGi at six months after starting empagliflozin but there was a significant reduction at one year. Improvement of hepatic fibrosis in patients with NAFLD may require at least one-year SGLT2 inhibitor treatment.

This study has several acknowledged limitations. First, this is a retrospective observational study without a control group, and the sample size is small. Second, lipid deposition in the liver was not evaluated using magnetic resonance. Third, the degree of hepatic fibrosis was not evaluated by histology but by reliable markers of hepatic fibrosis. Fourth, the combined use of medications for diabetes and dyslipidemia might bias these results. The influence of pioglitazone, a key medication for NAFLD, is interesting. Since only three patients used pioglitazone, formal assessment of its influence cannot be made. In this study, the NAFLD fibrosis score showed a likelihood of a decrement after one-year treatment, but the reduction did not reach statistical significance. Additional long-term observation may demonstrate a significant decrement in the NAFLD fibrosis score.

In conclusion, one-year empagliflozin treatment in patients with NAFLD complicated by type 2 diabetes mellitus resulted in significantly improved markers of hepatic inflammation, function and fibrosis. This is the first study reporting the effect of empagliflozin on NAFLD during a one-year treatment period. Since hepatic fibrosis progresses for several years, further longterm studies are necessary to confirm these preliminary results.

\section{CONFLICT OF INTEREST}

Author S. S. has received honoraria from AstraZeneca, Boehringer Ingelheim, Daiichi Sankyo, Kowa, Mitsubishi Tanabe and Takeda pharmaceuticals. Author M. O. received a research support from Takeda Pharmaceutical. Author M. O. also received 
honoraria from AstraZeneca, Boehringer Ingelheim, Daiichi Sankyo, Eli Lilly, Kyowa Hakko Kirin, Merck, Novo Nordisk, Mitsubishi Tanabe, Ono, Sanofi and Takeda pharmaceuticals. Other authors declare no conflicts of interest regarding this study.

\section{REFERENCES}

1. Watanabe S, Hashimoto E, Ikejima K, Uto H, Ono M, Sumida Y, Seike M, Takei Y, Takehara T, Tokushige K, Nakajima A, Yoneda M, Saibara T, Shiota G, Sakaida I, Nakamuta M, Mizuta T, Tsubouchi H, Sugano K, Shimosegawa T : Evidence-based clinical practice guidelines for nonalcoholic fatty liver disease/nonalcoholic steatohepatitis. J Gastroenterol 50 : 364-377, 2015

2. Eguchi Y, Hyogo H, Ono M, Mizuta T, Ono N, Fujimoto K, Chayama K, Saibara T : Prevalence and associated metabolic factors of nonalcoholic fatty liver disease in the general population from 2009 to 2010 in Japan : a multicenter large retrospective study. J Gastroenterol 47 : 586-595, 2012

3. Doycheva I, Cui J, Nguyen P, Costa EA, Hooker J, Hofflich H, Bettencourt R, Brouha S, Sirlin CB, Loomba R : Non-invasive screening of diabetics in primary care for NAFLD and advanced fibrosis by MRI and MRE. Aliment Pharmacol Ther $43:$ 83-95, 2016

4. Angulo P, Keach JC, Batts KP, Lindor KD : Independent predictors of liver fibrosis in patients with nonalcoholic steatohepatitis. Hepatology 30 : 1356-1362, 1999

5. Azuma K, Kadowaki T, Cetinel C, Kadota A, El-Saed A, Kadowaki S, Edmundowicz D, Nishio Y, Sutton-Tyrrell K, Okamura T, Evans RW, Takamiya T, Ueshima H, Curb JD, Abbott RD, Kuller LH, Kelley DE, Sekikawa A : Higher liver fat content among Japanese in Japan compared with non-Hispanic whites in the United States. Metabolism $58: 1200-1207,2009$

6. Fujita Y, Inagaki N : Update on the efficacy and safety of sodium-glucose cotransporter 2 inhibitors in Asians and non-Asians. J Diabetes Investig 10 : 1408-1410, 2019

7. Tahrani AA, Barnett AH, Bailey CJ : SGLT inhibitors in management of diabetes. Lancet Diabetes Endocrinol $1: 140-151,2013$

8. Jojima T, Tomotsune T, Iijima T, Akimoto K, Suzuki K, Aso Y : Empagliflozin (an SGLT2 inhibitor), alone or in combination with linagliptin (a DPP-4 inhibitor), prevents steatohepatitis in a novel mouse model of non-alcoholic steatohepatitis and diabetes. Diabetol Metab Syndr 8:45, 2016

9. Sattar N, Fitchett D, Hantel S, George JT, Zinman B : Empagliflozin is associated with improvements in liver enzymes potentially consistent with reductions in liver fat: results from randomised trials including the EMPA-REG OUTCOME (R) trial. Diabetologia 61 : 2155-2163, 2018

10. Takase T, Nakamura A, Miyoshi H, Yamamoto C, Atsumi $\mathrm{T}$ : Amelioration of fatty liver index in patients with type 2 diabetes on ipragliflozin : an association with glucose-lowering effects. Endocr J 64 : 363-367, 2017

11. Kuchay MS, Krishan S, Mishra SK, Farooqui KJ, Singh MK, Wasir JS, Bansal B, Kaur P, Jevalikar G, Gill HK, Choudhary NS, Mithal A : Effect of Empagliflozin on Liver Fat in Patients With Type 2 Diabetes and Nonalcoholic Fatty Liver Disease : A Randomized Controlled Trial (E-LIFT Trial). Diabetes Care 41 : 1801-1808, 2018

12. Komiya C, Tsuchiya K, Shiba K, Miyachi Y, Furuke S, Shimazu N, Yamaguchi S, Kanno K, Ogawa Y : Ipragliflozin Improves Hepatic Steatosis in Obese Mice and Liver
Dysfunction in Type 2 Diabetic Patients Irrespective of Body Weight Reduction. PLoS One 11 : e0151511, 2016

13. Ekstedt M, Franzen LE, Mathiesen UL, Thorelius L, Holmqvist M, Bodemar G, Kechagias S : Long-term follow-up of patients with NAFLD and elevated liver enzymes. Hepatology $44: 865-873,2006$

14. Seko Y, Sumida Y, Tanaka S, Mori K, Taketani H, Ishiba H, Hara T, Okajima A, Yamaguchi K, Moriguchi M, Mitsuyoshi H, Kanemasa K, Yasui K, Minami M, Imai S, Itoh Y : Serum alanine aminotransferase predicts the histological course of non-alcoholic steatohepatitis in Japanese patients. Hepatol Res 45 : E53-61, 2015

15. Hiraoka A, Kumada T, Kudo M, Hirooka M, Tsuji K, Itobayashi E, Kariyama K, Ishikawa T, Tajiri K, Ochi H, Tada T, Toyoda H, Nouso K, Joko K, Kawasaki H, Hiasa Y, Michitaka K: Albumin-Bilirubin (ALBI) Grade as Part of the Evidence-Based Clinical Practice Guideline for HCC of the Japan Society of Hepatology : A Comparison with the Liver Damage and Child-Pugh Classifications. Liver Cancer $6: 204-215,2017$

16. Kuno A, Ikehara Y, Tanaka Y, Ito K, Matsuda A, Sekiya S, Hige S, Sakamoto M, Kage M, Mizokami M, Narimatsu H : A serum "sweet-doughnut" protein facilitates fibrosis evaluation and therapy assessment in patients with viral hepatitis. Sci Rep $3: 1065,2013$

17. Yamasaki K, Tateyama M, Abiru S, Komori A, Nagaoka S, Saeki A, Hashimoto S, Sasaki R, Bekki S, Kugiyama Y, Miyazoe Y, Kuno A, Korenaga M, Togayachi A, Ocho M, Mizokami M, Narimatsu H, Yatsuhashi H : Elevated serum levels of Wisteria floribunda agglutinin-positive human Mac-2 binding protein predict the development of hepatocellular carcinoma in hepatitis $\mathrm{C}$ patients. Hepatology $60: 1563-1570,2014$

18. Abe M, Miyake T, Kuno A, Imai Y, Sawai Y, Hino K, Hara Y, Hige S, Sakamoto M, Yamada G, Kage M, Korenaga M, Hiasa Y, Mizokami M, Narimatsu H : Association between Wisteria floribunda agglutinin-positive Mac-2 binding protein and the fibrosis stage of non-alcoholic fatty liver disease. J Gastroenterol 50 : 776-784, 2015

19. Angulo P, Hui JM, Marchesini G, Bugianesi E, George J, Farrell GC, Enders F, Saksena S, Burt AD, Bida JP, Lindor K, Sanderson SO, Lenzi M, Adams LA, Kench J, Therneau TM, Day CP : The NAFLD fibrosis score : a noninvasive system that identifies liver fibrosis in patients with NAFLD. Hepatology 45 : 846-854, 2007

20. Sterling RK, Lissen E, Clumeck N, Sola R, Correa MC, Montaner J, M SS, Torriani FJ, Dieterich DT, Thomas DL, Messinger D, Nelson M : Development of a simple noninvasive index to predict significant fibrosis in patients with HIV/HCV coinfection. Hepatology $43: 1317-1325,2006$

21. Katsuki A, Sumida Y, Gabazza EC, Murashima S, Furuta M, Araki-Sasaki R, Hori Y, Yano Y, Adachi Y : Homeostasis model assessment is a reliable indicator of insulin resistance during follow-up of patients with type 2 diabetes. Diabetes Care 24 : 362-365, 2001

22. Kahl S, Gancheva S, Strassburger K, Herder C, Machann J, Katsuyama H, Kabisch S, Henkel E, Kopf S, Lagerpusch M, Kantartzis K, Kupriyanova Y, Markgraf D, van Gemert T, Knebel B, Wolkersdorfer MF, Kuss O, Hwang JH, Bornstein SR, Kasperk C, Stefan N, Pfeiffer A, Birkenfeld AL, Roden M : Empagliflozin Effectively Lowers Liver Fat Content in Well-Controlled Type 2 Diabetes : A Randomized, Double-Blind, Phase 4, Placebo-Controlled Trial. Diabetes Care $43: 298-305,2020$

23. Lai LL, Vethakkan SR, Nik Mustapha NR, Mahadeva S, Chan WK : Empagliflozin for the Treatment of Nonalcoholic 
Steatohepatitis in Patients with Type 2 Diabetes Mellitus. Dig Dis Sci 65 : 623-631, 2020

24. Inoue M, Hayashi A, Taguchi T, Arai R, Sasaki S, Takano $\mathrm{K}$, Inoue Y, Shichiri M : Effects of canagliflozin on body composition and hepatic fat content in type 2 diabetes patients with non-alcoholic fatty liver disease. J Diabetes Investig 10 : 1004-1011, 2019

25. Shibuya T, Fushimi N, Kawai M, Yoshida Y, Hachiya H, Ito S, Kawai H, Ohashi N, Mori A : Luseogliflozin improves liver fat deposition compared to metformin in type 2 diabetes patients with non-alcoholic fatty liver disease : A prospective randomized controlled pilot study. Diabetes Obes Metab 20 : 438-442, 2018

26. Sumida Y, Murotani K, Saito M, Tamasawa A, Osonoi Y, Yoneda M, Osonoi T: Effect of luseogliflozin on hepatic fat content in type 2 diabetes patients with non-alcoholic fatty liver disease : A prospective, single-arm trial (LEAD trial). Hepatol Res 49 : 64-71, 2019 